\title{
Plant 3D reconstruction based on LiDAR and multi-view sequence images
}

\author{
Jingwen Wu ${ }^{1,2}$, Xinyu Xue ${ }^{1 *}$, Songchao Zhang ${ }^{1}$, Weicai Qin ${ }^{1}$, Chen Chen ${ }^{1}$, Tao Sun ${ }^{1}$ \\ (1. Nanjing Institute of Agricultural Mechanization, Ministry of Agriculture, Nanjing 210014, China; \\ 2. Anhui Agricultural University, Hefei, China 230036, China)
}

\begin{abstract}
The 3D reconstruction of plant based on LiDAR is the main way to obtain the spatial structure of plant rapidly, nondestructive and all-weather. However, the influence of LiDAR instrument performance and field operation environment, the point cloud data obtained will lose the details of plant and reduce the accuracy of the model. In this study, the three-dimensional point cloud of plants generated based on multi-view sequence images was taken as a reference. The optimized Iterative Closest Point registration was adopted to calibrate the point cloud data from the LiDAR scanning to improve the detailed characteristics of the plants and establish a 3D model of plant. At the same time, according to the measured plant phenotype parameters (leaf length, leaf width, leaf area, plant height), the accuracy of 3D model was evaluated. The results showed that high accuracy of 3D reconstruction was obtained based on LiDAR and multi-view image sequence method. There was a good agreement between measured and calculated leaf area, leaf length, leaf width and plant height with $R^{2}>0.8$ for leaf area, RMSE $<1.0$ for leaf area, $R^{2}>0.85$ for leaf length, $R^{2}>0.95$ for leaf width. There was no significant difference for each phenotypic parameter between measured and calculated data (ANOVA, $P<0.05$ ). This method provides a technical reference for the research and application of LiDAR in fine modeling of field crops.
\end{abstract}

Keywords: LiDAR, multi-view sequence images, plant 3D reconstruction, accuracy evaluatione

DOI: $10.33440 /$ j.ijpaa.20180101.0007

Citation: Wu J W, Xue X Y, Zhang S C, Qin W C, Chen C, Sun T. Plant 3D reconstruction based on LiDAR and multi-view sequence images. Int J Precis Agric Aviat, 2018; 1(1): 37-43.

\section{Introduction}

Pesticide use is a crucial part of modern agricultural management. Under the context of precisoin agriculture, applying pesticide as efficiently as possible while minimzing the pesticide amount is deriable. To achieve this goal, knowledge of plant structure information is needed ${ }^{[1,2]}$. Three-dimensional (3-D) visualization of plant canopy is proposed in recent years to monitor plant growth and morphology ${ }^{[3]}$.

Currently, there are mainly two approaches for 3-D visualization of plant structure. One is virtual modeling including parametric and image methods ${ }^{[4]}$. Parametric modeling is to visually simulate long-term and large-scale field measurement parameters using geometric modeling. Combined with the L-system(Step by iteration through production rules, and use the regularity and self-similarity of natural plants to generate plant topologies) proposed by Lindenmayer ${ }^{[5]}$, a 3-D model to monitor wheat growth was established ${ }^{[6]}$. However, crop root growth is happening under the soil and therefore, it is not visible. Domestic researchers used the L-system to dynamically simulate the morphological structure and spatial distribution of the root growth

Received date: 2018-03-15 Accepted date: 2018-06-26

Biographies: Jingwen Wu, Master student, research interests: crop protection machinery and advanced pesticide application technology, Email: 2263322545@ qq.com; Songchao Zhang, Associate Scientist, research interests: precise pesticide spraying, Email: 51832898@qq.com; Weicai Qin, Assistant Scientist, research interests: plant protection and pest control, Email: 278886580@qq.com; Chen Chen, Assistant Scientist, research interests: electrostatic pesticide spray technology, Email: 270034737@qq.com; Tao Sun, Assistant Scientist, research interests: precision pesticide spraying, Email: 2260559763 @qq.com.

*Corresponding author: Xinyu Xue, $\mathrm{PhD}$, Senior Scientist, research interests: crop protection machinery and advanced pesticide application technology. Nanjing Research Institute for Agricultural Mechanization, Ministry of Agriculture Nanjing, 100 Liuying, Xuanwu District, Nanjing City, Jiangsu, 210014, China. Email: xuexynj@qq.com. processes of wheat ${ }^{[7]}$ (Wang, 2008), $\operatorname{corn}^{[8]}$ (Zhao, 2007), soybean ${ }^{[9]}$ (Zhong, 2008), and rice ${ }^{[10]}(\mathrm{Xu}, 2010)$. The image method modeling is to obtain the parameter sequence of the plant through image processing. According to the data information extracted from the image, the scholars realized the reconstruction of the crop canopy model ${ }^{[11-13]}$ and the morphological structure ${ }^{[14]}$. The equipment required for such a method is simple, and a high-precision plant model can be obtained, but the time required is long and the reconstruction speed is slow. The other is a real modeling method based on actual measurement data, including stereo vision and laser radar scanners, in which stereo vision is measured by multiple cameras and direct access to three-dimensional structural data such as fruit trees ${ }^{[15]}$ crops $^{[16]}$, etc. The module has a complex system structure and a large amount of calculation. Compared with virtual modeling and stereo vision modeling, LiDAR (Light Detection And Ranging) is an emerging technology in the field of measurement, which has fast, all-weather, efficient and accurate access to spatial topography information ${ }^{[17,18]}$ It has been gradually expanded from the military industry, applied to construction, forestry, agriculture and other fields. Escolà $\mathrm{A}$ et al. ${ }^{[19]}$ (2017) used a ground-based mobile laser scanner to obtain the point cloud data of olive trees. Comparing canopy volume estimates from lidar sensor readings with conventional estimations used in horticultural/horticultural studies, the results show that the correlation coefficient is between $r=0.56 \sim r=0.82$, which contributes to better orchard management. Sun et al. ${ }^{[20]}$ (2012) established a high-precision plant blade surface model based on point cloud data, which can quickly reconstruct the blade surface of the plant and identify the state of blade wilting and withering. Medeiros H et al. ${ }^{[21]}$ (2017) used Lidar to scan fruit trees from multiple angles. Based on the multi-view morphology and point cloud data of fruit trees, a 3D model was established to improve the pruning efficiency of winter dormant fruit trees.

LiDAR is highly affected by environmental factors in the field, 
and the crops strcuture itself..Combined with previous research methods and existing equipment, put forward a method of relate real modeling to virtual modeling, the image modeling with high accuracy, low cost, and plant color acquisition was selected in combination with LiDAR to compensate for the defects such as misplaced point data and incomplete information. The actual measurement based on LiDAR can reflect the real spatial structure information of plants, and the construction of multi-view sequence images can not only improve the detailed features of the plants, but also obtain the true color information of the plants. The rapeseed seedling stage (large leaf) was used as a reference, and the vertical and flat planting leaves (Green rose, evergreen) was used as the research subject. The model accuracies on different leaves structures were also evaluated.

\section{Materials and method}

This paper is divided into three parts, The first data acquisition part, including the acquisition of point cloud data based on multi-view sequence images, and the acquisition of LiDAR point cloud data. The second part is data processing, image-based point cloud data and LiDAR point cloud data registration, point cloud data denoising, Point cloud simplification. Part three, plant model reconstruction and model accuracy evaluation. The following picture shows the research process of this article.

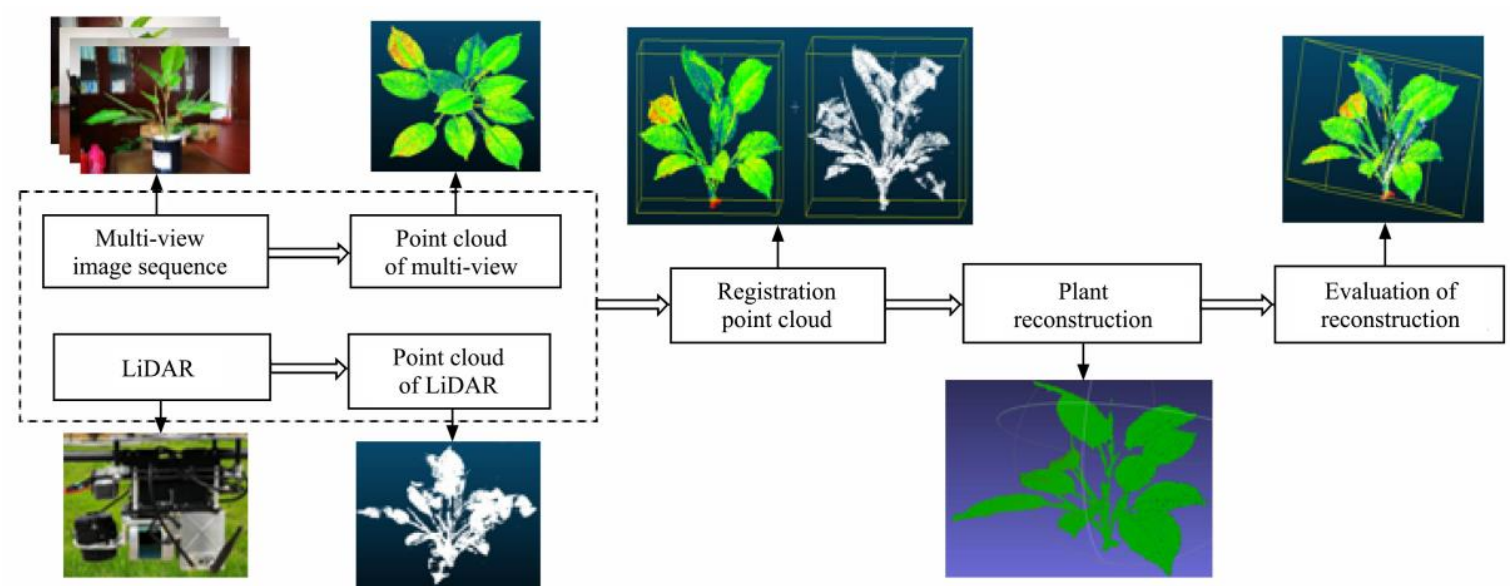

Figure 1 Process of 3-D plant reconstruction based on LiDAR and multiple view stereo method

\subsection{Point cloud data acquisition based on multi-angle stereo} vision

As mentioned earlier, green rose and evergreen was selected as the research subject. The plant was placed in an indoor environment without wind, strong light and electromagnetic field, or metal interference. Use the camera to shoot 25-40 images from top to bottom surrounding plants (Figure 2). It is notecible that overlapping is expected between adjacent sequence images (Figure 3 ).

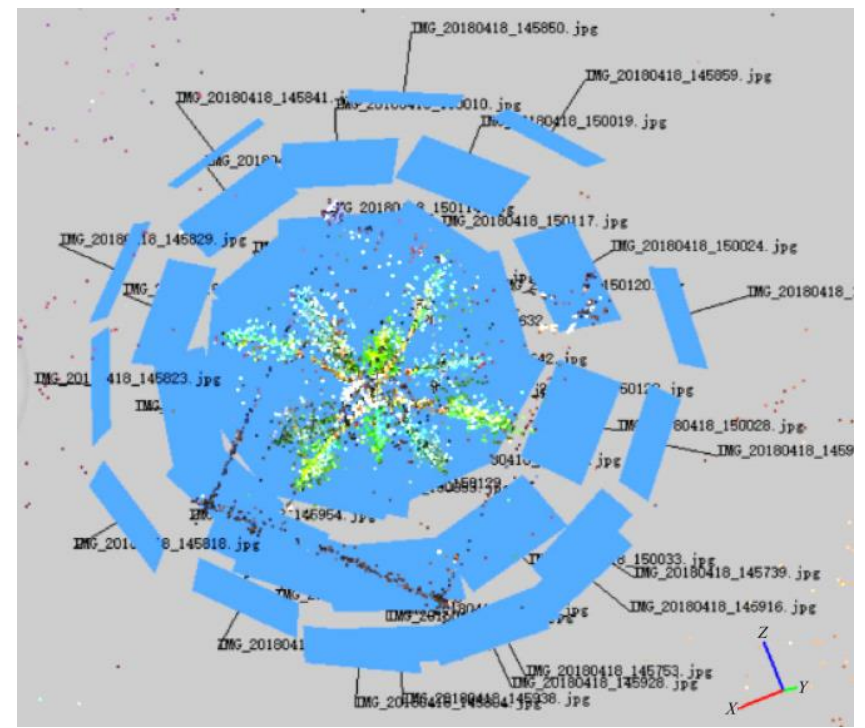

Figure 2 Camera positions around plant

Figure 4 illustrates the steps of extracting point cloud data from sequence image. The SIFT algorithm is used to search the image locations on all scales. The Gaussian differential function is used to identify the potential feature set of scale and rotation invariant in each image. The feature point set is matched in multiple images. Select two adjacent images with the most matching feature points to fit a fine model to determine the position and scale of the initial image pair ${ }^{[22,23]}$; then use the RANSAC algorithm to reconstruct the matched feature points, according to the calculated projection matrix, camera parameters, basic matrix values for bundling adjustment. Constantly adding new images, looking for new correspondences with matching 3D points that have already been matched, reusing RANSAC to calculate and adjusting clusters ${ }^{[24]}$. The above steps can use Agisoft software to process sequence images to obtain point cloud data based on multi-angle stereo vision.

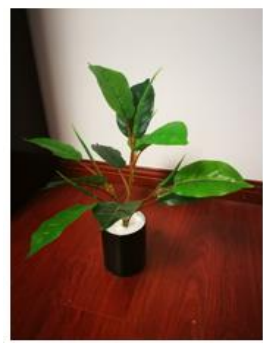

a. Image 19

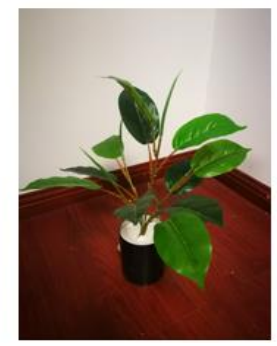

b. Image 20

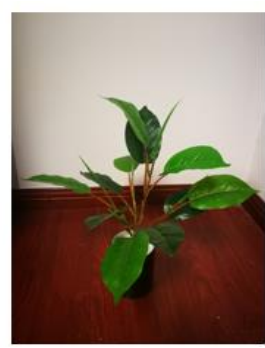

c. Image 21
Figure 3 Image examples of sequence images (take evergreen as an example)

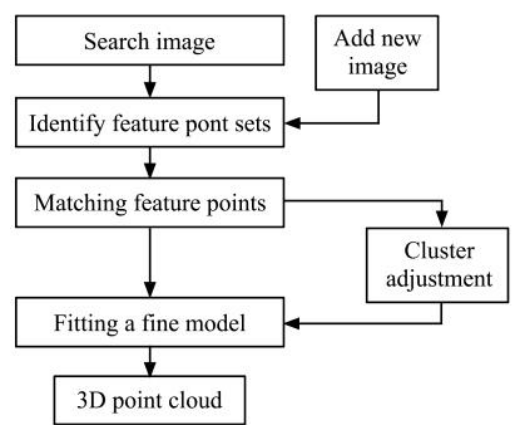

Figure 4 Extract point cloud data flowchart from image 


\subsection{Point cloud data collection based on laser scanner}

On April 11th, 2018, at the Baima Experimental Base in Jiangsu Province, an outdoor laser radar scanning was performed on evergreen and green rose. The Velodyne 3-D laser scanner was installed on a height-adjustable stand, and the stent was pushed around the plant to scan a circle. The resulting point cloud data records the spatial coordinates of each point of the plant.

\subsection{Point cloud registration}

Due to the limitations of the laser scanner's precision conditions and outdoor work, the leaves of plants are disturbed by the wind, resulting in the lack of information on the acquired point cloud data, the incomplete representation of the plant phenotype, and the inability to display the true color information of the plants. This paper proposes an algorithm for optimizing the ICP (Iterative Closest Point) to calibrate the lidar point cloud based on the point cloud data obtained from the image sequence. The specific method is described below.

The point cloud calibration mentioned above, also known as point cloud registration, is to find the rotation translation matrix between two point clouds and transform the Source Cloud and Target Cloud sets to the same coordinate system (here, the laser radar data is the target point cloud, and the image sequence data is the source point cloud), so as to obtain the complete point cloud set of data information, and the equation is expressed as follows:

$$
p_{t}=R \cdot p_{s}+T
$$

where, $p_{t}$ and $p_{s}$ refer to a pair of corresponding points in the target point cloud and the source point cloud, and $R$ and $T$ are the rotation translation matrix. The core idea of ICP point cloud registration is to use the initial $R$ and $T$ rotation translation matrix first, and use the nearest neighbor algorithm to find the optimal corresponding points $p_{t}^{i}$ and $p_{s}^{i}$ under the current conditions in $N_{p}$ pair points. Then, under the condition that the corresponding point is known, the objective function of $R$ and $T$ is minimized (which is, the sum of squared Euclidean distances between all corresponding points):

$$
f(R, t)=\frac{1}{N_{p}} \sum_{i=1}^{N_{p}}\left|p_{t}^{i}-R \cdot p_{s}^{i}-T\right|
$$

After obtaining the optimal $R$ and $T$ parameter values, some neighboring corresponding point positions will also change, so the last iteration needs to be repeated in turn until all corresponding points are traversed, and the position of the neighboring corresponding point no longer changes. Or the above objective function and the variation of $R$ and $T$ are less than a certain value, iteratively stopping ${ }^{[25]}$. At this point, it is considered to achieve the best registration effect (Figure 5).
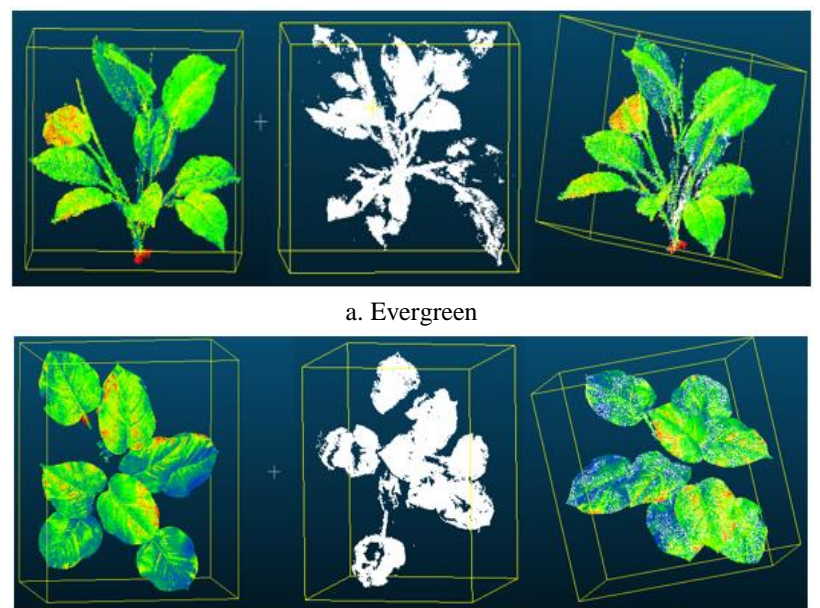

b. Green rose

Figure 5 Point cloud registration
As can be seen in the Figure 5, the calibrated point cloud compensates for the lack of information in the LiDAR point cloud, the local details are more complete, and the plant's true color information is covered on the point cloud.

\subsection{Point cloud denoising}

We know that no matter whether the point cloud data is acquired from the image or the point cloud data obtained by the laser radar, the noise point is easy to occur. The following explains the causes of noise generated by point cloud data obtained in two ways.

In the feature point matching process, due to the image itself (for example, blocking, shooting light, shooting the surrounding environment), it is unavoidable that points cloud have irregular data density, outliers, noise points, and so on. In addition, when using a three-dimensional laser scanner to work on plants, due to the influence of the scanner itself, external environment (such as wind), and human factors, the point cloud data acquired will generate a large number of noise points, outliers, and invalid points. These data component will reduce the speed of point cloud processing and the precision of morphological modeling.

Therefore, the acquired point cloud (Figure 6a) is denoised using a combination of manual and algorithmic methods. For the obvious and large amount of outliers were removed manually (Figure 6b), and for a small number of micro-offset noise points using algorithmic removal ${ }^{[26]}$ (Figure 6c). The result is a set of plant point cloud data with clear edges and smooth surfaces.
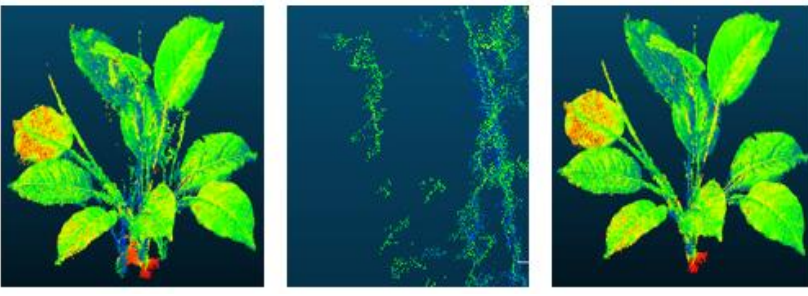

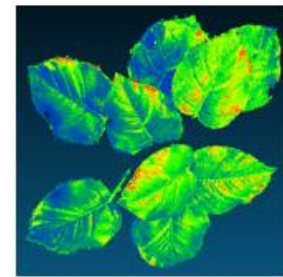

a. Green rose

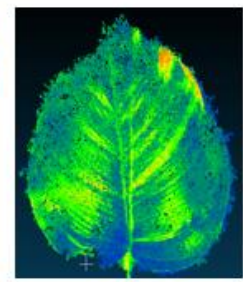

b. Amplification

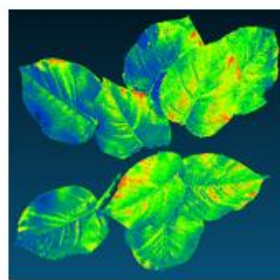

c. De-noising
Figure 6 Point cloud denoising before and after comparison chart (evergreen left, green rose right)

This article uses Statistical Outlier Removal filtering algorithm to denoise the point cloud. Let $P=\left\{P_{1}, P_{2}, \ldots P_{n}\right\}$ be the set of point clouds and $P\left(x_{i}, y_{i}, z_{i}\right)$ be any point in space. The algorithm first calculates the average distance from $N$ points to $K$ neighboring points by $K$-neighbor searching method, and then calculates the mean and standard deviation of $N * K$ distances. The standard deviation is defined as:

$\mu \pm \alpha \cdot \sigma$

In formula (1), $\mu$ and $\alpha$ are the mean and variance of $N * K$ distances, and $\sigma$ is the standard deviation. Points where the average distance is outside the standard deviation range are regarded as noise points, and the above calculations are repeated until all point clouds are traversed ${ }^{[27]}$. Select the average method $D\left(P_{i}\right)$ as the effect check parameter for noise removal, which is:

$$
D\left(P_{i}\right)=\frac{1}{K} \sum_{P_{j} \in N_{b}\left(P_{j}\right)}\left\|P_{i}-P_{j}\right\|
$$

Figures 7(a) and 7(b) show the $D\left(P_{i}\right)$ before removing the 
noise point and the $D\left(P_{i}\right)$ after the noise removal point (left

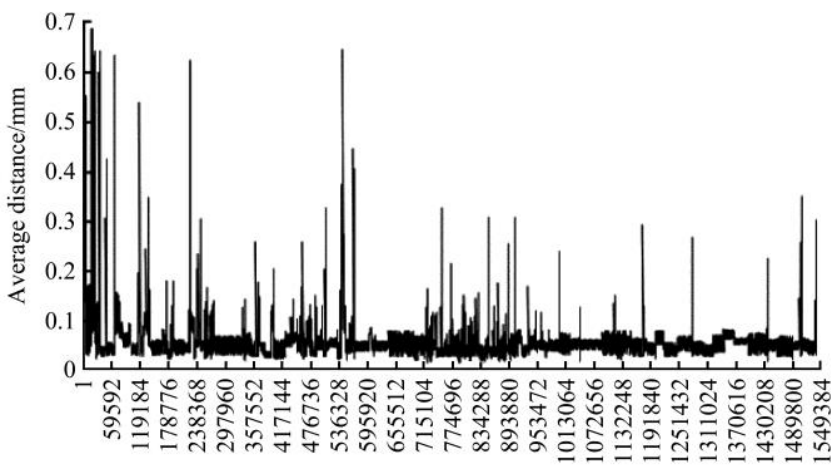

Center point evergreen, right green rose)

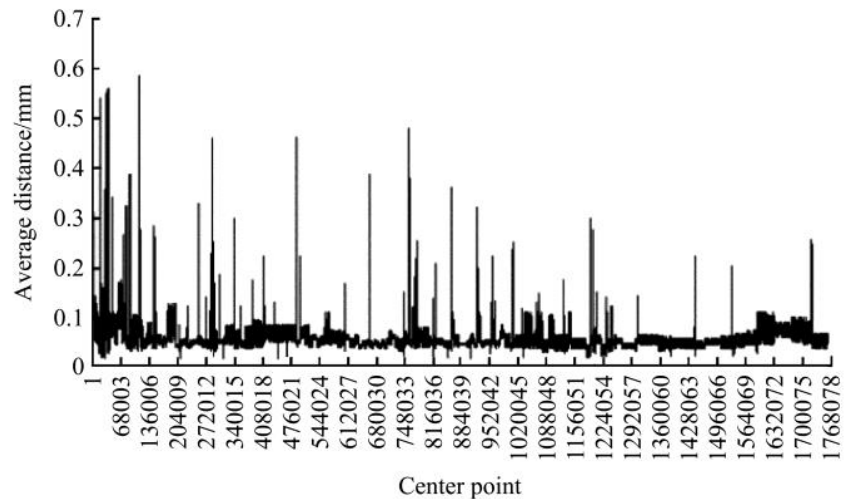

a. Before de-noising(left evergreen, right green rose)

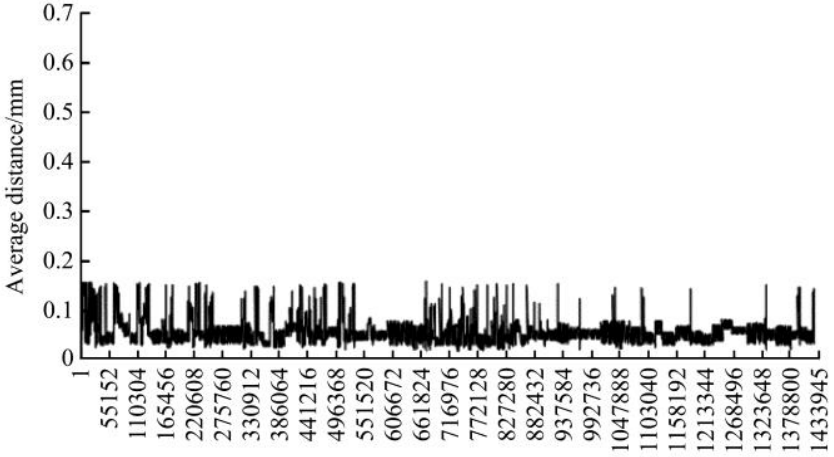

Center point

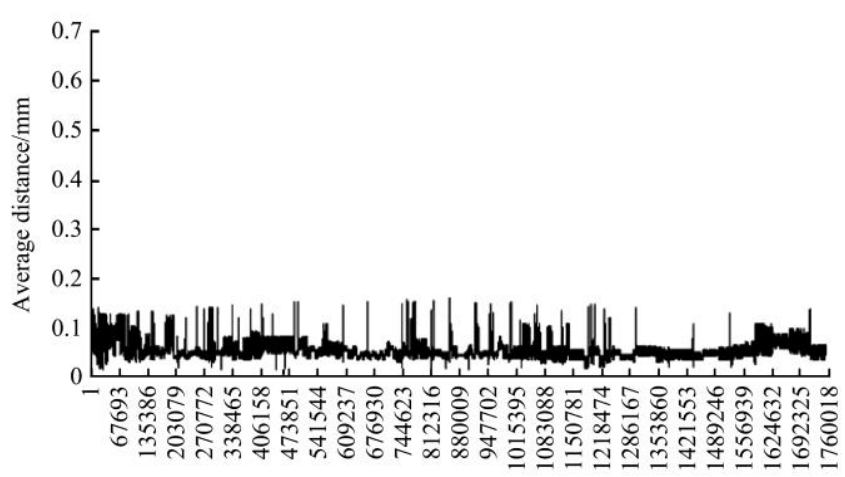

Center point

b. After de-noising(left evergreen, right green rose)

Figure 7 Comparision between values of $D\left(P_{i}\right)$ ( Before de-noising, De-noising)

\subsection{Point cloud simplification}

Although the registered point cloud data improves the structural phenotype information of the plant, the lidar data contained in it and the multi-angle stereoscopic point cloud data based on the image sequence will not only lead to a large amount of redundancy points in the data set, but the distance calculation of corresponding points in the registration process will also produce some glitches (also called noise). Registration enhance the accuracy and expands the amount of information, but also greatly increases the workload and storage space for data processing, slows down the rate of operations, and wastes computer resources.

According to the growth characteristics of the plant leaf, that is, the position where the tail of the plant is connected to the stem is the largest change in curvature. Along the leaf, the closer the leaf tip is, the smaller the curvature change is, and tends to be gentle. This study proposes an adaptive point cloud simplification method based on the curvature of a plant leaf. The steps are as follows.

(1) Uniformly divide the plant data containing $N$ points into a three-dimensional voxel grid of $10 \times 10 \times 10 \mathrm{~mm}^{3}$. After the grid is divided, place the point cloud in the corresponding grid and delete the grid that does not contain the point cloud data ${ }^{[28]}$.

(2) Calculates the Gaussian curvature and mean curvature for each point in the grid. Represent the local surface fitting equation in each grid as:

$$
Z(x, y)=a x^{2}+b x y+c y^{2}
$$

Suppose that any point $P_{i}$ in the grid has $k$ neighborhood points, and the coordinates of $P_{i}$ and its $k$ neighborhood points are brought into formula (5) to obtain the equations:

$$
B X=Z
$$

among them

$$
\begin{gathered}
B=\left[\begin{array}{ccc}
x_{0}^{2} & x_{0} y_{0} & y_{0}^{2} \\
x_{1}^{2} & x_{1} y_{1} & y_{1}^{2} \\
\vdots & \vdots & \vdots \\
x_{k}^{2} & x_{k} y_{k} & y_{k}^{2}
\end{array}\right] \\
X=(a, b, c)^{T} \\
Z=\left(Z_{0}, Z_{1}, \ldots Z_{k}\right)^{T}
\end{gathered}
$$

The linear least squares method is used to perform surface fitting on $P_{i}$ and $k$ neighborhood points. After the Hausdorff transformation method is used to obtain the coefficients $a, b$, and $c$ in the linear equations, the Gaussian curvature $K$ of $P_{i}$ can be calculated. The average curvature $H$ is:

$$
\begin{gathered}
K=4 a c-b^{2} \\
H=a+c
\end{gathered}
$$

After calculating the Gaussian curvature and average curvature of all points in the grid, according to different curvature values, divide the curvature threshold interval, set the curvature deviation as $\varepsilon$, if within a certain curvature interval, the point $P_{j}$ satisfies $\left|H_{j}-H_{i}\right| \leq \varepsilon$ for the base point $P_{i}\left(H_{j}\right.$ and $H_{i}$ are the average curvatures of points $P_{j}$ and $P_{i}$ ), delete point $P_{j}$, otherwise hold, and repeat the above calculation with $P_{j}$ point as the base point ${ }^{[29-31]}$.

(3) The calculation of step (2) is repeated for each point cloud data in each grid, and all plant point clouds are traversed, and the simplification effect is shown in Figure 8.

The adaptive point cloud simplification method based on the curvature of the plant blade is based on the criterion of the curvature change size to determine how much data is retained. Large curvature changes, data retention is more .From the verification of streamlining effect and the setting of the point cloud grid, the division of the curvature threshold interval is more accurate. By extractingthe geometric features of the plant, the 
data amount can be effectively reduced and the redundant points can be eliminated.
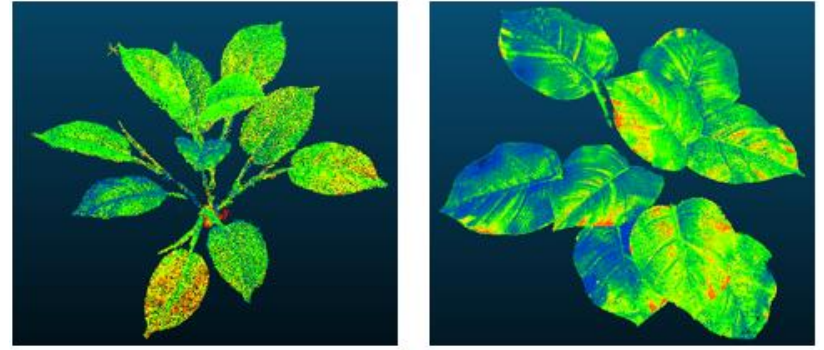

Figure 8 Simplification results (evergreen left, green rose right)

\section{Results and discussion}

\subsection{D model reconstruction results}

Gridding of point cloud data is an important method for 3-D modeling of plants. In this paper, Alpha-Shape algorithm is used to realize the reconstruction of 3-D models of streamlined point cloud data based on multi-view stereoscopic vision and LiDAR technology. By adjusting the Alpha value, it is possible to achieve contour extraction of leaves and stems of different sizes and shapes. According to the reduced point cloud density after processing and the query of empirical values in the literature, three Alpha values were set for the evergreen and green dill at the time of modeling. The evergreens were $0.086835,0.042690$ and 0.067384 , the green rose was $0.01859,0.04137,0.06973^{[32]}$.

It can be seen from the modeling effect of Figure 9 that when the Alpha value is set smaller, the details of the model are preserved better, such as the clear lines of the leaves. However, due to the fact that the point cloud is not evenly distributed on every part of the plant, "cavity" may appear in some parts, resulting in discontinuities in the center of the blade and stem, resulting in serious faults. With the increase of the Alpha value, the continuity of the plants after modeling is better and the morphology is more complete, but at the same time some of the plant's detailed information is lost.
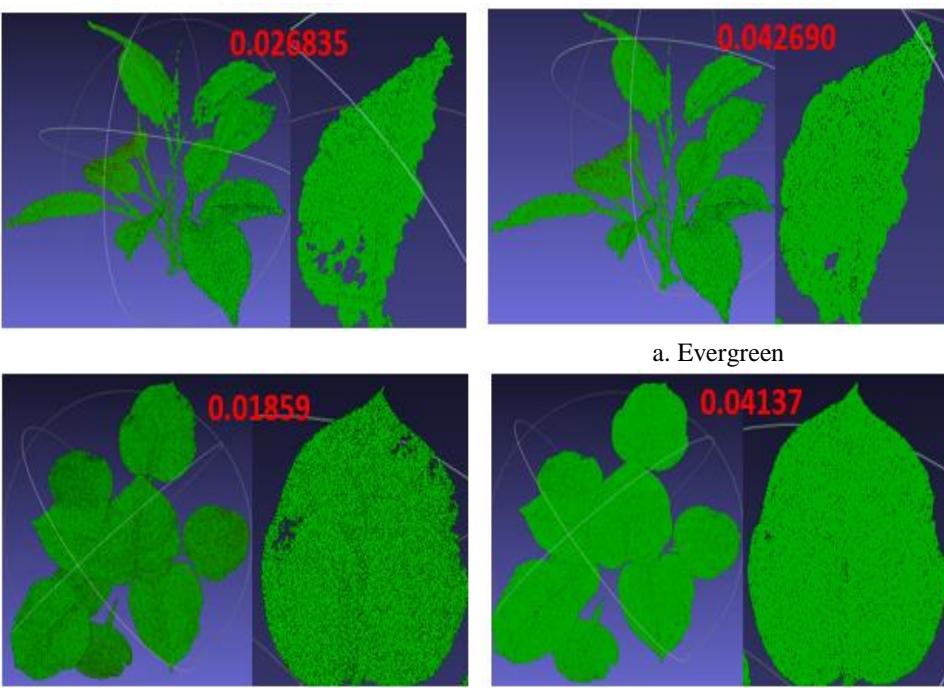

a. Evergreen

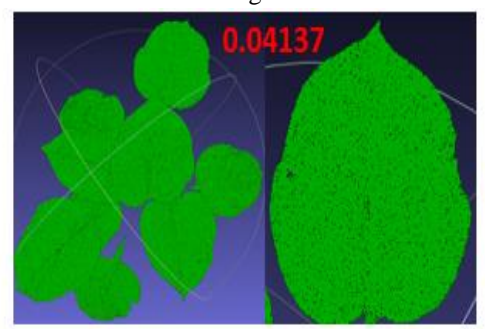

b. Green rose

Figure 9 3D reconstruction
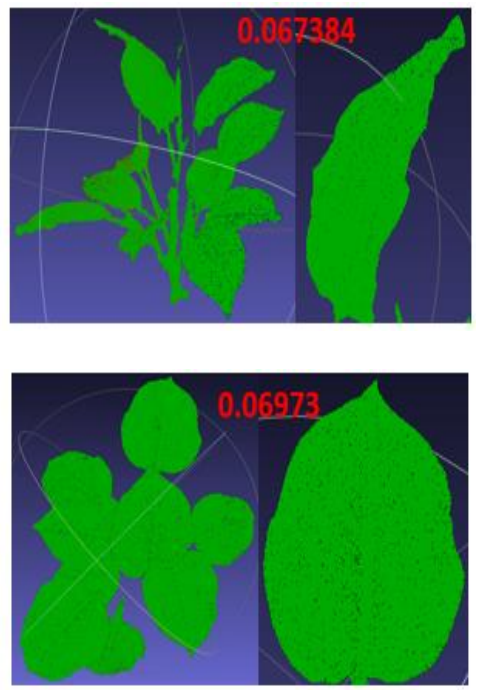

\subsection{Model accuracy assessment}

The Euclidean distance was used to measure the phenotypic parameters such as leaf length and leaf width of the extracted plants. Select the blade tip and blade base point from the three-dimensional reconstruction model of the leaf, and use this to segment the leaf. Vertically in the left and right direction, the middle coronal plane is constructed. The leaves are divided into two parts, front and back, and vertical in the front-rear direction. The middle sagittal plane is constructed. The leaves are divided into left and right sections. The left and right vertices are located on the sagittal plane and the vertices of the upper and lower sections. The farthest point of the coronal plane ${ }^{[33]}$ is calculated by the Euclidean distance from the left and right vertices to the sagittal plane and adding the distance from the top and bottom vertices to the coronal plane to obtain the leaf length and leaf width. The calculation of the leaf area of the plant is the sum of the area of the triangles in each curved surface of the reconstructed leaf.

From the method described above, the relative error RE, root mean square error RMSE, and determination coefficient $R^{2}$ were selected as the detection indexes by using basic statistical analysis methods. The leaves of green rose and evergreen plants were numbered from top to bottom. Euclidean distances were used to calculate the values of each parameter. The obtained models and measured parameters such as leaf length, leaf width, leaf area, and plant height were evaluated. Here,the manual measurement error of the steel measuring tares is as follows:

$$
\Delta= \pm(0.3+0.2 L) \mathrm{mm}
$$

where, $L$ is the length of the plant in metres (when the measured length is less than 1 meter, it is 1 ), and 0.3 is the fixed error of the steel tape ${ }^{[34]}$, and the measured errors of manually is $\pm 0.5 \mathrm{~mm}$.

Table 1 shows the relationship between calculated and measured leaf area. It is known from the table that the relative error between the model and the measured value of green rose and evergreen is less than 3\%, and the root mean square error RMSE is 0.79 and 0.49 , respectively. Although the coefficient of determination $\left(R^{2}\right)$ of green radish and evergreen was greater than 0.8 , there was a strong positive correlation, but the differences were large, 0.9229 and 0.8088 , respectively (Figure 10). The reason for this may be that the leaf space structure of the two plants is different. The leaf size of the green rose is larger, and the leaves exhibit a positional distribution that is approximately parallel to the horizontal plane and is less shaded. This type of plant morphological structure is easier obtained by the equipment.

The result of variance calculation showed that there was no significant difference between the measured and calculated leaf area $(P<0.05)$. It was proved that the three-dimensional plant model based on lidar and image sequences was able to reflect the leaf area attributes of real plants. 
Table 1 Relationship between calculated and measured leaf area

\begin{tabular}{cccc}
\hline Plant & $\begin{array}{c}\text { Relative } \\
\text { error/\% }\end{array}$ & $\begin{array}{c}\text { Root-mean-sqquare } \\
\text { error RMSE/cm }\end{array}$ & $\begin{array}{c}\text { Determination } \\
\text { Coefficient } R^{2}\end{array}$ \\
\hline Green rose & 0.98 & 0.79 & 0.9229 \\
Evergreen & 1.76 & 0.49 & 0.8088 \\
\hline
\end{tabular}

Figure 11 and Figure 12 is a correlation diagram obtained by comparing the leaf length and leaf width extracted from the three-dimensional model with the actual measured. It can be seen from the figure that the leaf width and leaf length calculated by the plant 3D model based on lidar and image sequences is in good agreement with the measured data. $R^{2}$ were all greater than 0.85 , and variance analysis showed no significant difference between the measured and calculated results $(P<0.05)$. Accurately reflects the true length and width of plant leaves. The results show that the true length and width of the leaves can be accurately determined.The heights of the plants were calculated to be $35 \mathrm{~cm}$ (green rose) and $39 \mathrm{~cm}$ (evergreen) respectively. The plant heights were $37 \mathrm{~cm}$ (green rose) and $42 \mathrm{~cm}$ (evergreen). The difference was small between the green rose $( \pm 2 \mathrm{~cm})$ and evergreen $( \pm 3 \mathrm{~cm})$.

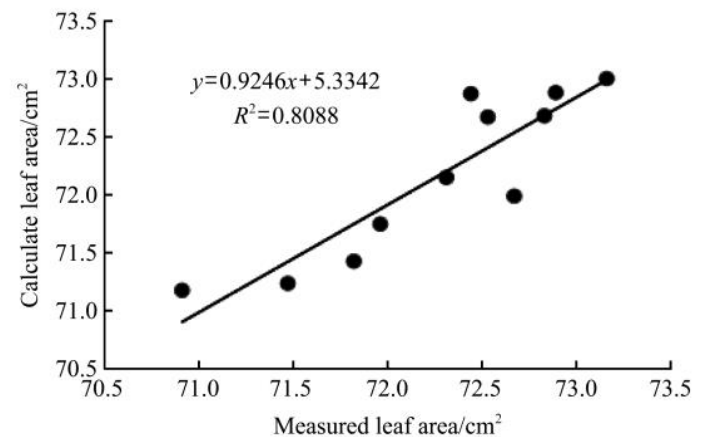

Figure 10 Relationship between leaf area results from calculate and measured (green rose on the left and evergreen on the right)
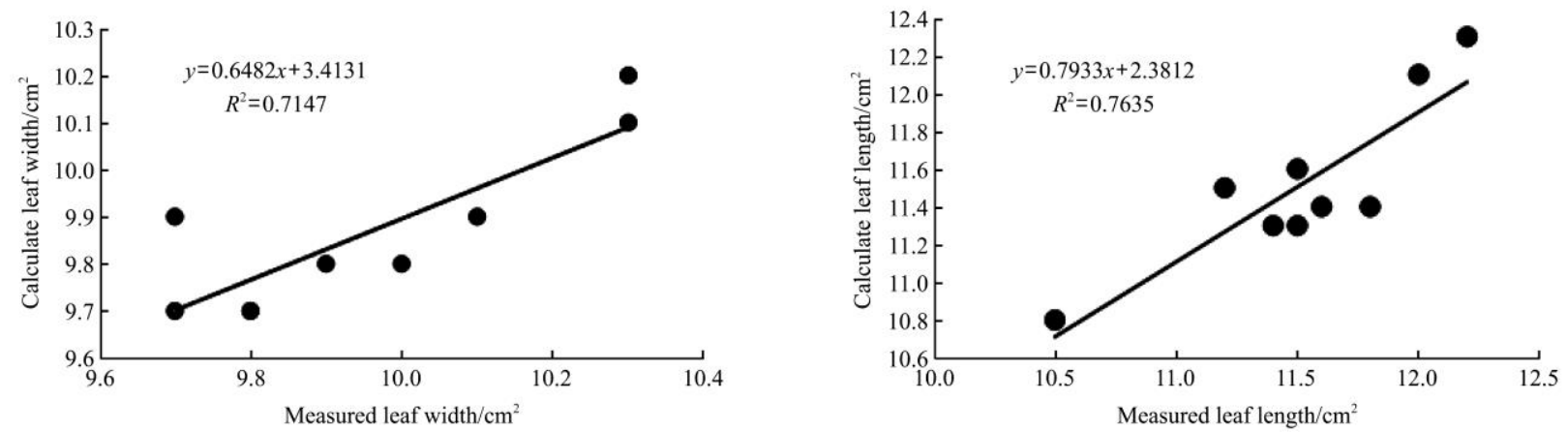

Figure 11 Relationship between green rose leaf width and length results from calculate and measured
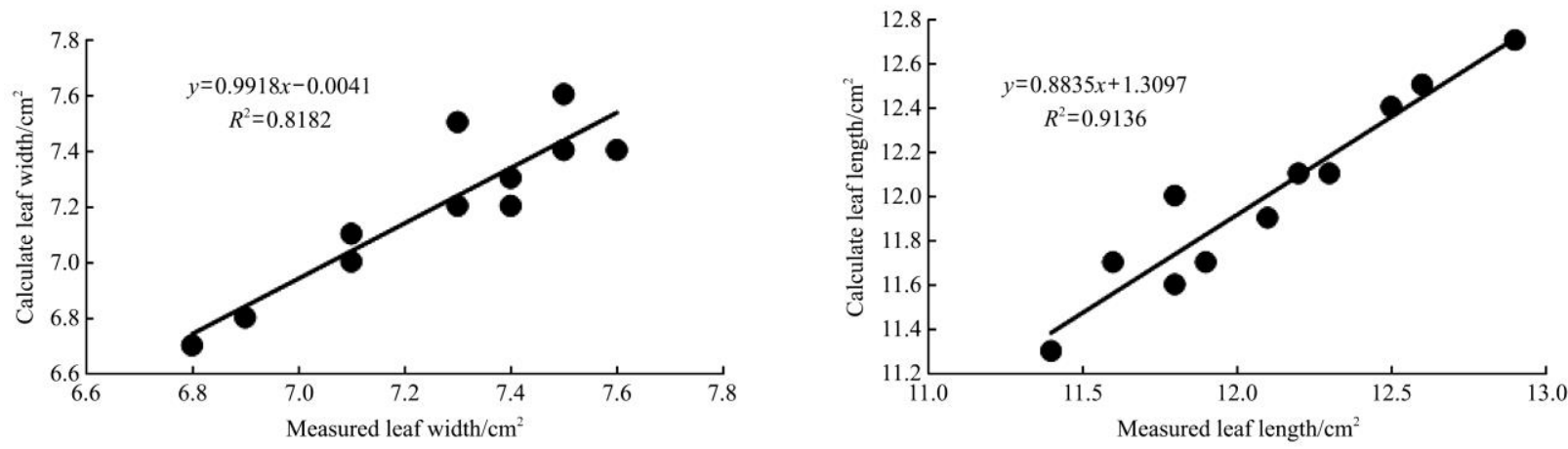

Figure 12 Relationship between evergreen leaf width and length results from calculate and measured

\section{Conclusions}

The conclusions of this study can be summarized as follows:

(1) The higher the quality of the point cloud based on the LiDAR plant scan test, the higher the model accuracy. The quality of the point cloud data obtained is not only related to the performance of the laser radar used, but also affected by factors such as outdoor weather, scanning methods, surrounding environment, and plant structure complexity. In windy conditions, the structure of the scanned object with many branches and obstructed around, the quality of the acquired point cloud data will be reduced. In addition, manual measurement errors can also unavoidably affect the accuracy of the model(measurement error is $0.5 \mathrm{~mm})$.

(2) The method of generating point clouds using multi-view sequence images is used to make up for missing information of point cloud data scanned by outdoor laser radar, providing a certain technical measure for the application of LiDAR in the research and application of fine crop modeling. Due to the influence of shooting light, the plant point cloud obtained based on the sequence image used in the text is more distorted than the real plant color, and the sequence image can be converted by using light irrelevant to eliminate the effect of illumination on the color extraction ${ }^{[35]}$.

(3) In this paper, the artificial green plants similar to the 
morphological structure of young rapeseed were selected to verify the feasibility of the three-dimensional modeling method based on Lidar and multi-view sequence images. In theory, the rape growing in the field environment is also suitable. Therefore, the focus of the next step is to conduct rapeseed field trials to verify the universality of this method.

(4) In the paper, the same modeling method was used to model three-dimensional blades with different morphological structure. The model accuracy results showed that the Alpha-Shape modeling algorithm works better for plants with large blades and less shelter between leaves.

(5) The method of generating point clouds by manually acquiring plant sequence images in this paper has the disadvantages of low automation and time-consuming increase. In the later stage, multi-technology integration can be used to build an automatic, real-time plant point cloud data acquisition device.

\section{Acknowledgement}

The authors gratefully acknowledge the financial support provided by the National Key Research and Development Plan (2017YFD0701000), the China Agriculture Research System (CARS-12); Collaborative Innovation Project of Chinese Academy of Agricultural Sciences (CAAS-XTCX2016006).

\section{[References]}

[1] Xue X Y, Lan Y B. Agricultural Aviation Applications in USA. Transactions of the CSAM, 2013; 44(5): 194-201.

[2] Zhai Changyuan, Zhu Ruixiang, Zhang Zuojing, et al. Status Analysis of Precision Pesticide Application Techniques. Journal of Agricultural Mechanization Research, 2010; 32(5): 9-12.

[3] Fang Hui, Du Pengpeng,Hu Lingchao, et al. VTK-based plant 3D morphological visualization and registration. Transactions of the CSAE, 2013; 29(22): 180-188.

[4] Liu Dan, Zhu Yeping, Liu Hailong, et al. Research Progress on 3D Plant Visualization. Journal of Agricultural Science and Technology, 2015; 17(1): 23-31.

[5] Lindenmayer A. Mathematical models for cellular interactions in development. Journal of Theoretical Biology, 1968; 18(3): 280-299.

[6] Fournier C, Andrieu B, Ljutovac S, et al. ADEL-Wheat: a 3D architectural model of wheat development// International Symposium on Plant Growth Modeling, Simulation, Visualization and Their Applications, 2003.

[7] Wang Meili, He Dongjian. Visualized Simulation of Wheat Roots Based on L-System. Journal of Agricultural Mechanization Research, 2008; (3): 36-39.

[8] Zhao Chunjiang, Wang Gongming, Guo Xinyu, et al. 3D visualization of corn root system based on interactive framework model. Transactions of the CSAE, 2007; 23(9): 1-6.

[9] Zhong Nan,Luo Xiwen,Qin Qin.Modeling and visualization of three-dimensional soybean root system growth based on growth functions. Transactions of the CSAE, 2008, 24(7): 151-154.

[10] Xu Qijun, Tang Liang, Gu Dongxiang, et al. Architectural parameter-based three dimensional modeling and visualization of rice roots. Transactions of the CSAE, 2010; 26(10): 188-194.

[11] Renatopratademoraes F, Witoldf K. Three-dimensional digital model of a maize plant. Agricultural \& Forest Meteorology, 2010; 150(3): 478-488.

[12] Lordan, J., Pascual, M., Fonseca, F., Montilla, V., Papio, J., Rufat, J., et al. An image-based method to study the fruit tree canopy and the pruning biomass production in a peach orchard. HortScience, 2015; 50(12): 1809-1817.
[13] Rufat, J., Villar, J. M., Pascual, M., Falguera, V., \& Arbonés, A. Productive and vegetative response to different irrigation and fertilization strategies of an Arbequina olive orchard grown under super-intensive conditions. Agricultural Water Management, 2014; 144, 33-41.

[14] Liu Gang, Zhang Xue, Zong Ze, et al. 3D Reconstruction of Strawberry Based on Depth Information. Transactions of The Chinese Society for Agricultural Machinery, 2017; 48(4): 160-165.

[15] Biskup B, Scharr H, Schurr U, et al. A stereo imaging system for measuring structural parameters of plant canopies. Plant Cell \& Environment, 2007, 30(10): 1299-1308.

[16] Ivanov N, Boissard P, Chapron M, et al. Computer stereo plotting for 3-D reconstruction of a maize canopy. Agricultural \& Forest Meteorology, 1995, 75(1-3): 85-102.

[17] Su Wei, Guo Hao, Zhao Donglin, et al. Estimation of Actual Leaf Area of Maize Based on Terrestrial Laser Scanning. Transactions of the Chinese Society for Agricultural Machinery, 2016; 47(7): 345-353.

[18] Andújar D, Rueda-Ayala V, Moreno H, et al. Discriminating crop, weeds and soil surface with a terrestrial LIDAR sensor. Sensors, 2013; 13(11): $14662-75$.

[19] Escolà A, Martínez-Casasnovas J A, Rufat J, et al. Mobile terrestrial laser scanner applications in precision fruticulture/horticulture and tools to extract information from canopy point clouds. Precision Agriculture, 2017; 18(1): 1-22.

[20] Sun Zhihui, Lu Shenglian, Guo Xinyu, et al. Surfaces reconstruction of plant leaves based on point cloud data. Transactions of the CSAE, 2012; 28(3): 184-190.

[21] Medeiros H, Kim D, Sun J, et al. Modeling Dormant Fruit Trees for Agricultural Automation. Journal of Field Robotics, 2017; 34.

[22] Lowe D G. Objective recognition from local scaleinvariant features Proc.int.conf.on Comput.vision, 1999, 2.

[23] Lowe D G. Distinctive Image Features from Scale-Invariant Keypoints// International Journal of Computer Vision, 2004; 91-110.

[24] Hu Pengcheng, Guo Yan, Li Baoguo, et al. Three-dimensional reconstruction and its precision evaluation of plant architecture based on multiple view stereo method. Transactions of the CSAE, 2015; 31(11): 209-214.

[25] Aiger D, Mitra N J, Cohenor D. 4-points congruent sets for robust pairwise surface registration. Acm Transactions on Graphics, 2008; 27(3) $1-10$.

[26] Zhang Weijie, Liu Gang, Guo Cailin, et al. Apple Tree Leaf Three-dimensional Reconstruction Based on Point Cloud. Transactions of the CSAE, 2017; 48(Supp.): 103-109.

[27] Rusu R B, Marton Z C, Blodow N, et al. Towards 3D Point cloud based object maps for household environments. Robotics \& Autonomous Systems, 2008, 56(11):927-941.

[28] Aiger D, Mitra N J, Cohenor D. 4-points congruent sets for robust pairwise surface registration. Acm Transactions on Graphics, 2008; 27(3) $1-10$.

[29] Yuan Hua, Pang Jiankeng, Mo Jianwen. Research on Simplification Algorithm of Point Cloud Based on Voxel Grid. Video Engineering, 2015; 39(17): 43-47.

[30] Li Kezhen, Lou Xiaoping, Lv Naiguang. Research on data simplification for point cloud in surface reconstruction. Journal of Beijing Institute of Machinery, 2009; 24(1): 17-20.

[31] Magid E, Soldea O, Rivlin E. A comparison of Gaussian and mean curvature estimation methods on triangular meshes of range image data. Computer Vision \& Image Understanding, 2007; 107(3): 139-159.

[32] Liu Rui, Liu Ting, Dong Runru, et al. 3D modeling of maize based on terrestrial LiDAR point cloud data. Journal of China Agricultural University, 2014; 19(3): 196-201.

[33] Anthony P, Xavier S, Scott B, et al. A novel mesh processing based technique for 3D plant analysis. Bmc Plant Biology, 2012; 12(1): 1-13.

[34] GB/T 2443-2011, Steel measuring tares.

[35] Ran N L, Filin S, Eizenberg H. Plant growth parameter estimation from sparse 3D reconstruction based on highly-textured feature points. Precision Agriculture, 2013; 14(6): 586-605. 